\title{
Metabolic syndrome is associated with aggressive colorectal tumour phenotype in males only
}

\author{
L. A. Healy ${ }^{2}$, A. M. Ryan ${ }^{2}$, J. Howard ${ }^{1}$, D. Flannery ${ }^{1}$, B. Mehigan ${ }^{1}$, R. Stephens ${ }^{1}$ and J. V. Reynolds ${ }^{1}$ \\ Department of Surgery ${ }^{1}$, Department of Clinical Nutrition ${ }^{2}$, St James's Hospital and Trinity College Dublin
}

The metabolic syndrome(MetS) is the clustering of risk factors including central obesity, hypertension, and raised plasma glucose, triacylglycerols and HDL cholesterol. Its presence may induce metabolic perturbations, alterations in cell signalling pathways and oxidative stress which could promote tumour development or growth. MetS has been associated with increased risk of colorectal cancer and mortality ${ }^{(1)}$.

The aim of this prospective observational study of colorectal cancer patients was to examine the incidence of MetS and its relationship to tumour pathology in an Irish population. Patients underwent a metabolic and nutritional assessment prior to treatment. MetS was defined according to the International Diabetes Federation definition ${ }^{(2)}$

One hundred and fourteen colorectal cancer patients (53\% male: $47 \%$ female) were recruited with a median age of 69 years (range 36-88). MetS was diagnosed in $42 \%$ patients, with a similar incidence in males (54\%) and females (47\%), which exceed the population norms reported at $21 \%{ }^{(3)}$. MetS was significantly associated with a more advanced pathological stage $(P=0.018)$, nodal involvement $(P=0.004)$ and extramural vascular invasion $(P=0.012)$, and these effects were significantly $(P<0.05)$ more marked in males.

We report for the first time a high prevalence of MetS and central obesity in a cohort of Irish patients with colorectal cancer. MetS seems to be associated with a more aggressive tumour phenotype, and the implications of this with respect to prevention and treatment require further study.

1. Cholangelo LA et al. (2002) Cancer Epidemiol Biomarkers Prev 11, 385-391.

2. Alberti KGMM et al. (2006) Diabet Med 23, 469-480.

3. Waterstone DF et al. (2009) Ir J Med Sci 178(2): 161-6. 